\title{
Einschränkung der Nationalen Gestaltungsmöglichkeiten Angesichts der Wachsenden Globalisierung und die Rolle der Zivilgesellschaft für Mögliche Gegenstrategien*
}

\author{
Friedrich Müller ${ }^{1}$
}

Zusammenfassung: Der Nationalstaat ist im Verlauf der Globalisierung in seiner Wirkung stark eingeschränkt worden, vor allem wirtschafts-, finanz-, sozial- und umweltpolitisch. Er wurde aber deswegen nicht überflüssig. Denn zugleich behält er unverzichtbare politische Funktionen bei der Verteidigung von Republik, Demokratie und Rechtsstaat und besonders auch bei der nationalen, supranationalen und transnationalen Entwicklung der Menschen- und Bürgerrechte. Er sollte sich dabei mit den Widerstandsbewegungen aus der Zivilgesellschaft strategisch verbünden, die in Richtung auf ein öffentliches Gewissen im Weltmaßstab und auf eine globale civil society arbeiten.

Stichworter: Globalisierung. Nationalstaat. Zivilgesellschaft.
Abstract: The nation-state has been severely restricted in the course of globalization in its effect, especially in economic, financial, social and environmental policy. But even so it became unnecessary. Because the nation state retains at the same time crucial political functions in the defense of the republic, democracy and the constitutional state, as well it mainly retains human development at the national, supra-national and trans-national level - and civil rights. Then it should join strategically to resistance movements in civil society that are working towards a public conscience on a global scale and a global civil society.

Key words: Globalization. Nation state. Civil society.

\footnotetext{
* (C) Friedrich Müller

1 Professor de Direito Constitucional, Filosofia do Direito e Teoria Geral do Direito na Universidade de Heidelberg - Alemanha.E-mail: muellerfedja@arcor.de.

Recebido em: 15/07/2012.
}

Revisado em: 31/07/2012.

Aceito em: 08/09/2012. 


\section{Einführung}

Der Term „national“ im Thema meint nationalstaatlich. Der Nationalstaat ist heute in seiner Wirkung deutlich eingeschränkt, behält aber unverzichtbare Funktionen bei. Im Europa des 15. bis zum 19. Jahrhundert entstanden, stellt er sich als politisch und rechtlich souveräne Organisation auf einem bestimmten Territorium dar. Für die Entwicklung von Republik, Rechtsstaat und Demokratie, von Sozialstaat, Menschen- und Bürgerrechten bot er historisch und bietet er noch heute den Rahmen, den (verfassungs-) rechtlich gesicherten Raum. Der demokratische nationale Verfassungsstaat erscheint als das leistungsfähigste aller Systeme der bisherigen Politikgeschichte: gerade auch für Reformen, für eine Sozialpolitik, die auf den Abbau von Ungleichheit abzielt, auf gesellschaftliche und politische Inklusion aller. (BRUNKHORST; KETTNER, 2002; WEHLER, 2001; NGUYEN, 2002; MÜLLER, 2001 und 2003)

Was den Nationalstaat seit einigen Jahrzehnten - und zunehmend - in Frage stellt, ist das Ende der nach außen abschließbaren Organisationsform. Die zentral wichtigen Entscheidungen sind auf sie nicht mehr beschränkbar: planetare Ökologie, weil das Ökosystem der Erde im Ganzen auf einen Kollaps zutreibt; weltweit spekulativ auf sich selbst „referierende" Finanzmärkte, die vom realen Wirtschaften abgekoppelt sind; Produktion, Distribution und Arbeitsmarkt, die dabei sind, die traditionellen Grenzen hinter sich zu lassen. Mit anderen Worten: das wirklich Wichtige ist nicht mehr territorial und auch immer weniger material begrenzbar. So verliert der Nationalstaat seine traditionellen Grundlagen: statt des Staatsgebiets ein globales Spielfeld, statt Staatsvolk und Staatsnation eine zunehmend individualistisch differenzierte Gesellschaft, die de facto auf eine Weltgesellschaft hinausläuft. Nicht nur alle Kommunikationen, sondern auch die Grundfunktionen wie Wirtschaft, Politik, Wissenschaft werden weltweit „simultan“ erfüllt. Eine unbegrenzt de-zentrierte Formation dieser Art ist von nationalen Machthierarchien nicht mehr einzufangen: Entmaterialisierung in der Produktion, Entterritorialisierung im Steuerungsapparat, Entnationalisierung in der Vorstellung von „Souveränität". So wird das zentrale Strukturierungsinstrument des Nationalstaats, die Rechtsordnung, nicht nur von formellem Völkerrecht und internatio- 
nal geltenden Menschenrechten modifiziert sowie in Europa von supranationalen Normen überwältigt, sondern sogar von einem immer umfangreicheren Corpus von informellem de-facto-Weltrecht (wie: Codes des elektronischen Handels, Geschäftsbedingungen multinationaler Konzerne) ergänzt, vor allem aber umgangen, unterlaufen; durch eine ohne Staat, ohne zentrale Gesetzgebung und Gerichtsbarkeit rein tatsächlich funktionierende, transnationale, privatnützige „Justiz des Stärkeren“ (dazu z. B. MÜLLER, 2003)

\section{Nationalstaat und Globalisierung}

Allein schon deswegen ist davor zu warnen, den Nationalstaat als Konzept und als Mittel praktischer Politik über Bord werfen zu wollen. Demokratie und Rechtsstaat benötigen sein Potenzial in der Résistance gegen die Zumutung vom „Recht“" des Stärkeren. Und er wird als - wenn auch inzwischen relativierter - Rahmen für soziale Reformpolitik und für die Aktivität der Zivilgesellschaft immer dringender gebraucht. Der europäische Nationalstaat, geboren aus den Religionskriegen der beginnenden Neuzeit, hatte Formen entwickelt, den Zusammenprall von Ideologien und sogar den Widerspruch zwischen Kapital und Arbeit im Prinzip friedlich zu beantworten. Diese Widersprüche lösen sich jetzt, zusammen mit der herkömmlichen Dominanz des Nationalstaats, nicht etwa auf; sie bestehen, in nur teilweise veränderter Form, in der Weltgesellschaft fort. Sie verlangen inhaltliche Antworten - und auf dem Weg zu diesen vor allem auch politische Einrichtungen, normierte Verfahren und prozedurale Standards. Diese müssen transnational und global entwickelt, aber auch national weiter entwickelt werden. (dazu BRUNKHORST; KETTNER, 2000; BRUNKHORST, 2002; LUTZ-BACHMANN, 1999; ROSAS, 2000; THÜRER, 2000; HABERMAS, 1998a, 1998b und 1999; SCHMALZ-BRUNS, 1999; MÜLLER, 2003)

Das, was wir semantisch verniedlichend „Globalisierung“ nennen, ist eben nicht nur eine gleichsam bloß technische wirtschaftliche Integration der Welt; eine komplexe weltweite Arbeitsteilung, ein rasantes betriebs- und volkswirtschaftliches Umstrukturieren und das Anwachsen 
gegenseitiger ökonomischer Abhängigkeit. Es ist vor allem ein neuartiges Gewalt- und Machtspiel, eine aus dem Hintergrund steuernde nicht-demokratische planetare Exekutive aus IWF, Weltbank, WTO, OECD und den G 8-Gipfeln. Es ist ein ebenso abstraktes wie brutales Anwenden der ökonomischen Modelle und der wirtschaftspolitischen Ideologie der reichsten westlichen Industrieländer auf die Gesellschaften der so genannten Peripherie. Die Mondialisierung ist im Kern eine mondiale Monetarisierung; die Globalisierung eine solche der Gesetze des Kapitals: Profitmaximierung für wenige durch Angebotspolitik, „Flexibilisierung“ der Lohnabhängigen, durch Sozialabbau und Privatisierung, niedrige Steuern, Steuerfreiheit für „Standort"wahl, freie Wechselkurse, Liberalisierung der Finanztransfers - kurz durch Abbau des Systems von Bretton Woods. Nach diesem wurden Devisen getauscht, um damit Handel und reale Investitionen zu finanzieren - also auf einem Feld, das immer schon das der Politik und der demokratischen Verantwortung der einzelnen Staaten gewesen war. Die globale Deregulierung, das Markenzeichen des Turbokapitalismus, gab auch diesem System von 1944 den Todesstoß mit dem Ergebnis, dass um die Wende 1999/2000 bereits 98 \% der täglich floatenden Kapitalmenge von rund eineinhalb Billionen US \$ spekulativ waren (1971 waren es nur $10 \%$ gewesen, $90 \%$ hatten sich im älteren Wirtschaftsmodell noch auf reales Investivkapital bezogen).

Die sozio-ökonomischen Folgen dieser globalen Politik sind verheerend: die Einkommensschere klafft immer weiter auseinander, 20 Prozent der Menschheit genießen inzwischen 90 Prozent der Güter; die reichsten 200 Weltbürger verfügen über ein Vermögen, das dem jährlichen Einkommen der Hälfte der Menschheit entspricht (über eine Billion US $\$$ ); eine Milliarde Personen lebt im Wohlstand, eine Milliarde in grausamem Elend, vier Milliarden vegetieren am Existenzminimum.

All das ist inzwischen bekannt, und es ist hier nicht das Thema. Thema ist die Tatsache, dass die Gewählten nicht mehr (das Wichtige) entscheiden und dass die Entscheider nicht gewählt sind; Thema ist, dass Wirtschaftspolitik in Sozial- und Verteilungspolitik eingebettet und das Ganze demokratisch verantwortet werden muss, dass aber unter den heutigen Bedingungen sozialstaatliche Modelle und solche der gemischten Wirtschaft wie „Ordoliberalismus“ und ,soziale Marktwirtschaft“, 
wie „New Deal“" und „Great Society“, die Normen und Regeln brauchen, selbst dereguliert und tendenziell zum Verschwinden gebracht werden. (MÜLLER, 2001)

Das Ganze ist so offenkundig, dass es auch außerhalb der wissenschaftlichen Kritik, außerhalb der Beteiligten am Porto-Alegre-Prozess und der „Alterglobalisierer" nicht mehr gut versteckt werden kann: schon bei einer Umfrage aus Anlaß der WTO-Konferenz in Seattle glaubten, aller organisierten Meinungspolitik zum Trotz, nur noch $37 \%$ der US-Amerikaner in der unteren Hälfte der Einkommenspyramide an die Vorteile der Globalisierung; dagegen waren unter den Wohlhabenderen $63 \%$ zutreffend davon überzeugt, die globale Wirtschaft helfe gerade ihnen.

\section{Zivilgesellschaft und Globalisierung}

Die Frage richtet sich angesichts all dessen auch auf mögliche Gegenstrategien und auf die Rolle der Zivilgesellschaft in deren Rahmen. Daher soll an einigen Punkten noch näher angedeutet werden, worin die bisher gegebene Diagnose begründet ist.

(1) Zur Entgegensetzung „,Markt vs. Staat": Die Vertreter des „reinen Marktes“ verherrlichen diesen und verteufeln die staatliche Steuerung. Das ist historisch sehr fragwürdig; stabil waren immer vor allen anderen die gemischten Systeme. Ferner gibt es Korruption und Überbürokratisierung nicht nur in Staatsapparaten, die Wirtschaft wimmelt geradezu davon. Korruption und Delinquenz sind ,im Markt“ gängige Münze, mit immer noch steigender Tendenz. Die Zahl der hierher zählenden Skandale in allen G-8-Staaten ist kaum mehr zu überblicken. Die Privatwirtschaft scheut demokratische Kontrolle, weil sie sehr viel Kriminelles zu verbergen hat; ihre konzertierte Polemik gegen den Staat ist ein durchsichtiges Manöver. Die Massenmedien, welche diese Polemik verbreiten, sind nicht mehr unabhängig; sie sind inzwischen das Eigentum großer Mischkonzerne, denen es nicht um faire Information geht, sondern um Macht. Das ,duale System 'von Radio und Fernsehen z.B. in Frankreich und Deutschland (hier ist es sogar pluralistisch) ist eine notwendige Antwort hierauf - eine der zentralen Mitwirkungsmöglichkeiten der Zivilgesellschaft, von denen hier die Rede ist. 
Das öffentliche Interesse, das bonum commune, wird - zwischen Staat und Markt - nur von staatlicher Seite vertreten. Probleme der Verteilungspolitik brauchen den Staat. Der Anspruch der Demokratie erfordert es, wirtschaftliche Vorgänge in soziale einzubetten - inzwischen auf nationaler und auf internationaler Ebene. Sonst machen staatsfreie Marktvorgänge die demokratische Verantwortung der Verfassungsstaaten endgültig zur Farce. Die sogenannten Marktkräfte sind aber weder Naturgesetze noch höherrangige Regeln von Geschichte und Gesellschaft; sie sind Menschenwerk, parteiisch und interessengebunden, sind privatnützig und blind profitbesessen. Dass sie dem oberflächlichen Blick als funktionierend erscheinen können, liegt nur daran, dass realiter weithin Systeme der gemischten Wirtschaft bestehen; auch die neoliberalen Lautsprecher, die am hartnäckigsten jede staatliche Kontrolle denunzieren, haben deshalb noch nie staatliche Subventionen (aus Steuergeldern) verschmäht.

Die Zivilgesellschaften müssen - inhaltlich gesehen - auf staatlicher Mitwirkung und demokratischer Kontrolle bestehen, auf viel konsequenterer Verfolgung von Wirtschaftsverbrechen, auf dem Beseitigen der Steuerparadiese, auf Schuldenerlass und gerechtem Handel, auf Besteuerung von Kapital- und Spekulationserträgen und auf einer pluralistischen Reorganisation der meinungsmachenden Massenmedien. Das geschieht über politischen Druck auf die nationalen Regierungen und auf - gleichfalls politisch veranlassten - Druck durch diese auf inter- und transnationale Entscheidungszentren. Die Zivilgesellschaft muss dabei Hand in Hand mit dem Nationalstaat arbeiten, den Druck allerdings aus eigener Initiative erzeugen. (MÜLLER, 2001, S. 68 ff., 86 ff.; 2003, S. 83 ff., 115 ff.)

(2) Zur Frage Globalisierung und Demokratie: In der Neuen Wirtschaft braucht der Unternehmer den Lohnabhängigen nicht mehr unbedingt. Zunehmend riesige Gruppen von Menschen werden überflüssig, werden de facto wirtschaftlich, dann sozial, dann kulturell und schließlich am Ende dieser Kettenreaktion sogar noch rechtlich/politisch exkludiert. Das gilt zunehmend auch für die entwickelten kapitalistischen Gesellschaften, und die Vierte Welt der Überzähligen ist schon eine globale Erscheinung. Die traditionelle Demokratie, d.h. das repräsentative Modell, unterliegt angesichts dessen selber einer systemischen Exklusion: wachsende Teile der Gesellschaft, also der kommunikativ zu integrierenden 
Einzelnen und Gruppen, kommen ihr abhanden - und damit ihre Grundlage. Für Länder in Prozessen demokratischer Transformation (wie Südafrika oder Brasilien und viele andere) kommen die dafür unentbehrlichen Potenziale der Zivilgesellschaft gar nicht erst ins Spiel. Schadet die Globalisierung schon den entwickelten Demokratien, so kann ihre bisherige Wirkung für Transitionsgesellschaften fatal werden. Politische Rechte (in the books) brauchen soziale Voraussetzungen, um (in action) ausgeübt werden zu können. Und auch globale Akteure wie der IWF üben sich darin, nationale Demokratien zu umgehen, auszutricksen, unter Druck zu setzen - Joseph Stiglitz, der es wissen muss, hat das erschreckend beschrieben. Der Fonds, demokratisch so wenig verantwortlich wie Weltbank, OECD oder WTO, handelt dementsprechend verantwortungslos, hat die Verhandlungsmacht monopolisiert, unterminiert den - soweit schon vorhanden - mühsam funktionierenden demokratischen Prozess. Den Rahmen für das Ineinander nationaler, regionaler, inter- und transnationaler Normierungen, Orientierungen, Markt- und Machtchancen bildet immer weniger der demokratische Nationalstaat mit seiner ,souveränen' Rechtsordnung. Gegenkräfte können hier nur aus der Zivilgesellschaft kommen. Durch diese Vorgänge schrumpft aber zugleich die Entscheidungsmacht nationaler Parlamente und Regierungen in wichtigen Fragen. Die Folgen der Globalisierung verhindern in manchen Fällen das Entstehen vitaler Demokratien, bremsen in vielen Fällen Demokratisierung und schwächen bestehende demokratische Systeme - indem sie diesen wachsende Teile ihres demos entziehen und ferner den gewählten Instanzen zentrale Entscheidungen aus der Hand nehmen. Die Politikmüdigkeit, die seit langem alarmierenden Prozentsätze ständiger Wahlenthaltung in den G-8-Ländern werden inzwischen als chronisch resigniert hingenommen, statt als hochgradig alarmierend bearbeitet zu werden. Mobiles Kapital auf einem staat(en)losen Markt überspielt gewählte Parlamente und Regierungen, auch durch die übliche Erpressung mit „Standortwechsel“", nicht zuletzt in vitalen Fragen des Umweltschutzes. All das beraubt die Zivilgesellschaft, so weit sie aktiv ist, vielfach des nationalen Partners. Es verweist sie darauf, mit ihren schwachen Mitteln - vernetzte Kommunikation, symbolische Aktion, Sachvorschläge, Protest - auf künftige transnational wirksame Mittel demokratischer Kontrolle hinzuarbeiten. 
Ein Weltstaat erscheint dabei nicht wünschenswert, schon gar nicht unter demokratischen Aspekten; und die Idee eines Weltparlaments sollte zugunsten engerer, dafür aber auch konkreterer Pläne zu den Akten gelegt werden: entscheidende Stärkung des Europäischen Parlaments und auch der UN-Vollversammlung in ihrer Stellung gegenüber dem Sicherheitsrat. Zumindest im Rahmen regionaler Wirtschaftsblöcke, die nicht auf Domination angelegt sind, sondern auf Kooperation (EU, Mercosul; nicht dagegen die von den USA fast schon obsessiv betriebene gesamtamerikanische Freihandelszone), sollte die traditionelle Kompetenz des Nationalstaats zu reformistischer Politik fortgeführt werden: ein ständiges Politikziel des sozialen Ausgleichs, eine hinreichend faire materielle Umverteilung und das Sichern der Gleichheit von Bildungschancen. Die neue brasilianische Präsidentschaft hat sich solche Ziele gesetzt - wenn auch auf einer (von neoliberalen Vorgängern geerbten) äußerst ungünstigen Ausgangslage.

(3) Zur ökonomischen Grundlage der hier untersuchten Phänomene:

Die Stichwörter heißen bekanntlich „Ende des Industriezeitalters“, „Übergang von der Industrie- zur Wissensgesellschaft“, ,digitale Revolution“, „New Economy“. Das alte Modell bestand aus technisch-industrieller Produktion und demokratischer Regulierung über Rechtsvorschriften. Seine Hauptakteure waren Kapital, Arbeit und Staat; die Gewerkschaften hatten Einfluss und drängten auf eine als reformistisches Politikziel durchaus vorhandene Umverteilung. Die realen Stützen bildeten stetiges Wachstum von Wirtschaft und Arbeitsproduktivität auf der einen und zunehmende Massenkaufkraft auf der anderen Seite. Das änderte sich seit den 60er Jahren des 20. Jahrhundert im technischen, seit Mitte der 70er Jahre im politischen Bereich. Die technische Seite (tendenzielle Immaterialität der Wertschöpfung, Deterritorialisierung, Vernetzung) ist kaum zu beeinflussen, auch nicht durch die Zivilgesellschaft. Darüber ist aber die politische nicht zu vergessen: der Abschied von den traditionellen Parametern und die globale Deregulierung waren massive ideologische Entscheidungen (Flexibilisierung der Wechselkurse, Aufgabe der Kontrollen des Kapitalverkehrs, Sozialabbau, Privatisierung, Sturz des System von Bretton Woods, Unternehmenskonzentration, usw.). Sie können auch wieder z.T. modifiziert, z.T. durch neue Formen überholt werden: Kapitalkontrollen, 
Erträgnisbesteuerung, ein den heutigen Problemen angepasstes „Bretton Woods II", eine internationale Kartellbehörde im Rahmen der Weltarbeitsorganisation, usw. Indem die Nationalstaaten diese Aufgaben anpacken, füllen sie zum Teil wieder ihre frühere Rolle als steuernde Instanzen aus - jetzt allerdings zunehmend auf dem inter- und transnationalen Spielfeld, auf dem sie sich wieder energischer als Akteure zurückmelden sollten. Das erfordert allerdings den Mut zur politischen Résistance gegen den Hegemon, z.B. durch Beharren auf einer Demokratisierung im Rahmen des IWF oder der WTO. Der Übermacht dieser Zentralagenturen, zusammen mit Weltbank, OECD und G-8-Gipfeln, teilweise Außenstellen der Regierung in Washington, ist nur durch Politik zu begegnen; und diese bezieht Projekte und Energie nur aus der je nationalen und aus der sich bildenden globalen Zivilgesellschaft. Dass diese Vorgänge äußerst mühsam sind, ist kein Gegenargument: dieselbe Sequenz ,zivilgesellschaftliche Anstöße $\rightarrow$ ihnen entsprechende Politik der Nationalstaaten $\rightarrow$ Ergebnisse in der internationalen Arena“ prägte, nur als Beispiel, den Weg zu den neueren Menschenrechtspakten, zur Antifolter- und Antilandminenkonvention, zur Einrichtung des Ständigen Internationalen Strafgerichtshofs.

\section{Partizipative Demokratie und Globalisierung}

Der globale Markt und seine Agenten geraten bereits in Legitimationsnot. Die sozial und politisch aktiven Bürger kämpfen für eine verstärkt partizipative Demokratie sowie transnational für verzweigte, beweglich verbundene kollektive Handlungsformen. Diese Formen sind nicht mehr an „Nation“ oder den Nationalstaat gebunden, sind Formen staatloser Demokratisierung (Vgl. BRUNKHORST; KETTNER, 2002; BRUNKHORST, 2003; MÜLLER, 2003). Ohne sie wird es nicht möglich sein, gegen perverse Effekte dieser Art von Globalisierung wirksam einzutreten. Zu den überkommenen ungelösten Problemen (die sich verschärfende Ungleichheit, das ökologische Desaster, Gewalt und Militarismus, nichtstaatlicher und staatlicher Terrorismus) kommt die neuartige Aufgabe, transnationale und globale Mobiles aus Netzen, Institutionen und beginnenden Normierungen zu erarbeiten. Rahmenkompetenzen eines 
Weltrechts und globaler Konstitutionalismus sind dabei Stichworte für die im engeren Sinn juristischen Ziele. Träger all dieser Bewegungen - Protest, symbolische Aktion, konstruktive Vorschläge, geglückte Provokationen der Justiz (um diese in Gang zu setzen) - ist seit der Endphase des 20. Jahrhunderts die globale Galaxie demokratischer, menschenrechtlicher und alter-mondialistischer Nichtregierungsorganisationen geworden: soziales Weltgewissen, Störfaktor für die Routine der Unterdrückung und Ausbeutung, für die faktisch bestehende undemokratische Weltherrschaft, Generator intelligenter Alternativen; dezentrierte Globalisierung „,von unten", ohne Herrschaft auszuüben, über Lobbying und Druck auf die öffentliche Meinung wirksam, kurz: in der sich konsolidierenden Rolle einer global civil society. Sie arbeitet, durch Staatsgewalt nicht gestützt, friedlich und meinungsbildend für Werte des Gemeinwohls (global public goods), die - von den gleichen Menschenrechten für Alle bis zur globalen Ökologie - auf Pluralität und Universalität abzielen. Ihre Aktivität stützt sich nicht zuletzt selber auf nationale und internationale Menschen- und Bürgerrechte. Sie liefert hochqualifiziertes Expertenwissen, leistet nachhaltige Sacharbeit, hält die Konflikte bewusst und den lebenswichtigen politischen Widerstreit aufrecht - Grundfunktionen pluralistischer Demokratie. Dieses sich abzeichnende transnationale Volk als Akteur einer politischen und direkt agierenden Kultur globalen Widerstands steht in seiner demokratischen Legitimation, verglichen mit den traditionellen politischen Parteien, Gewerkschaften, Kirchen und sonstigen pressure groups, entgegen aller neoliberalen Polemik sehr achtbar da. Sein Zentrum liegt nicht in der Institution, sondern in der Aktion, nicht in formalen Dispositiven, sondern in den verteidigten und angestrebten Inhalten; es ist nicht „repräsentativ“ im ritualisierten Sinn der bürgerlichen Systeme, sondern graswurzeldemokratisch.

Dabei geht es nicht darum, „die“ Nichtregierungsorganisationen im allgemeinen hochgestimmt zu bejahen, gar emphatisch zu feiern. Unter ihnen finden sich alle Formen innerer Ordnung, von absolutistischer Hierarchie bis zu kaum regulierter Demokratie und spontaner Organisation. Bei Nichtregierungsorganisationen aus den G 7-Ländern sind, auf dem Terrain der Dritten und der Vierten Welt, ferner Erscheinungen wie übergroße Bürokratisierung oder Demonstration von Überlegenheit festzustel- 
len, zum Teil nur schwer leicht vermeidbar. Die Tätigkeit von NGOs aus den reichen Ländern verschleiert nicht selten auch die wirklichen Machtund Gewaltverhältnisse, ähnlich der kirchlichen Mission im historischen Kolonialismus. Das hier Gesagte stützt sich vielmehr auf die Rolle der autochthonen, der in den betroffenen Ländern selbst und typischerweise im Widerstand gegen politische und/oder wirtschaftliche Gängelung und Übermacht arbeitenden Organisationen.

In Ländern mit diktatorischen oder sonst autoritären Machtzentren wird diese Art informeller Demokratisierung vielleicht noch einmal entscheidend werden. Auch intern (noch) nicht demokratische, sondern beispielsweise klientelistische Einzelgruppen tragen wenigstens zu einem gewissen Plualismus der civil society bei. Wenn ausreichend große Teile der Bevölkerung auf diese Weise organisierbar werden, können sich Machteliten, jedenfalls auf längere Sicht, nicht ohne Zusammenwirken mit ihnen halten. Auch kann bei allmählich höherem Grad der Konsolidierung dadurch zunächst in einzelnen Ländern etwas wirksam werden, was ich „Parallelverfassungen“" nenne.

Diese sich national, aber auch schon zunehmend außer- und transstaatlich organisierende Kultur sozialer und politischer Résistance wird durchaus wahrgenommen; der Gründer und Gastgeber der Davoser Wirtschaftsgipfel hat schon im Jahr 2000 einräumen müssen, ,wir können nicht gegen die Gesellschaft wirtschaften“. Die sich globalisierende Zivilgesellschaft hat, mit anderen Worten, schon den Umriss jenes globalen öffentlichen Raums (aus Kommunikation und Interaktion) geschaffen, der für demokratische Reformen unverzichtbar ist; den öffentlichen Raum, in dem sich die nationalen Grundrechtsgarantien und die der Internationalen Menschenrechte politisch auswirken können - politisch im Sinn von Demokratie als der Staatsform der Inklusion - was wiederum die gesellschaftliche Realität gleichheitlich gewährter Menschen- und Bürgerrechte voraussetzt, also den legitimierenden Raum für rechts- und sozialstaatliche Reformpolitik angesichts einer exkludierenden Praxis der liberalen Zweidrittel (im entwickelten Weltzentrum)- bis Einzehntelgesellschaften (in der Peripherie). 
Fragt man, über das bisher Gesagte hinaus, nach weiteren spezifischen Formen, die das Handeln dieser neuartigen civil society annehmen kann, so stößt man auf avancierte Gestaltungen partizipativer Demokratie. Diese sind vielfältig und würden ein selbständiges Referat erfordern. Ich nenne hier nur zwei vielsagende Beispiele:

Die nationale wie die bisherige globale Zivilgesellschaft, deren Handlungsformen ich beschrieben habe, sind jeweils eine ,schwache Öffentlichkeit"; d.h. geeignet, auf formelle Entscheidungen zwar Einfluss zu nehmen, ohne aber solche selbst fällen zu können. Das ändert sich auch dann noch nicht, wenn die Betroffenen in der Selbstverwaltung gesetzliche Mitwirkungsrechte auf Information, Einspruch, gerichtliche Gegenwehr etwa im Planungs- oder im Umweltrecht eingeräumt erhalten. Es ändert sich dagegen, sobald entweder über solche Vorhaben verbindliche Sachplebiszite möglich sind; oder sobald der von Porto Alegre aus entwickelte und inzwischen weltweit als vorbildlich diskutierte Beteiligungshaushalt eingeführt wird. Dieser kann über Stadt- und Verkehrsplanung auf alle Fragen der Infrastruktur und der für den Alltag wichtigen öffentlichen Funktionen ausgedehnt werden - eine demokratisierende Verschiebung innerhalb der im engeren Sinn politischen Entscheidungsvorgänge aufgrund einer nunmehr starken Öffentlichkeit (Zu den Begriffen, m. Nw., BRUNKHORST, 2002 und 2003; zu den Erfahrungen mit dem von Porto Alegre ausgehenden Beteiligungshaushalt beispielsweise. (GENRO; DE SOUZA, 1998; FEDOZZI, 1999; ABERS, 2000; GRET; SINTOMER, 2002; MÜLLER, 2003, S. 110 ff.)

Das zweite Beispiel betrifft die Sachplebiszite. So lange das Volk nur die Gesetzesinitiative hat und die politische Oligarchie mit dieser dann nach Belieben verfährt, oder das Volk lediglich über von der politischen Elite vorformulierte Vorlagen durch Referendum abstimmen darf, bleibt es in der Position einer schwachen Öffentlichkeit. Stark wird diese erst durch die rechtlich obligatorische Kombination von Volksbegehren und Volksentscheid - sei es in jedem Fall, sei es im Fall der Ablehnung einer populären Initiative durch das Parlament. Diese Volksgesetzgebung kann auch mit dezentraler Gesetzgebung verbunden werden; und zwar auf Gebieten, die den Alltag Aller betreffen, etwa im Tarif- und Arbeits- 
recht, im Mietrecht, im Umwelt- und Verbraucherschutz ( $\mathrm{Zu}$ den einzelnen Formen: MAUS, 1992).

\section{Schlussfolgerungen}

Zur theoretischen Strukturierung der hier entwickelten Überlegungen deute ich noch zwei Punkte an: Einmal kann nur so die Polarisierung zwischen globalem Turbokapitalismus und dem überwunden werden, was Jean Baudrillard die „Singularitäten“" nennt. Nur diese, schreibt er, könnten sich dem universal gewordenen System der Domination entgegensetzen, wobei namentlich der Terrorismus zu diesen Singularitäten zählt. Die weltweite Résistance einer sich gleichfalls globalisierenden Zivilgesellschaft entwickelt nun aber nach ihren Inhalten und Handlungsweisen einen verallgemeinerungsfähigen Gegenpol.

Und zweitens: Dieser Widerstand der Zivilgesellschaft hat nur in einer doppelten Bewegung reale Erfolgsaussichten; wer will, kann darin eine Realdialektik erkennen. Zum einen ist der Nationalstaat als Form schon zu sehr geschwächt und ist die Zivilgesellschaft als globale noch nicht stark genug, um über die Rebellion als Ensemble von Singularitäten hinauszukommen. Die Zivilgesellschaft als jeweils nationale muss die Staaten zum Widerstand motivieren, sie im Widerstand orientieren; damit diese dann international, in Europa auch supranational und im ganzen global mit den Mitteln des internationalen Rechts (Abkommen, Pakten, Konventionen, Politik im Rahmen der UN und der anderen genannten Einrichtungen) agieren. Das wiederum wird die als solche informelle globale civil society auf ihrem Weg zu Gegenstrategien stärken, die dem globalen Gegendruck noch einmal werden standhalten können.

\section{Referenzen}

ABERS, Rebecca Neaera. Inventing local democracy. Grassroots Politics in Brazil. London: Lynne Rienner Publishers, 2000. 
BRUNKHORST, Hauke. Globale demokratie ohne staat? Schwache Öffentlichkeit, starke Öffentlichkeit - globaler Konstitutionalismus. 2003. (unveröffentlicht)

. Solidarität. Von der Bürgerfreundschaft zur globalen Rechtsgenossenschaft. Frankfurt/ Main: Suhrkamp, 2002. BRUNKHORST, Hauke; KETTNER, Matthias (Hrsg.). Globalisierung und Demokratie. Wirtschaft, Recht, Medien. Frankfurt/ Main: Suhrkamp, 2000.

FEDOZZI, Luciano. Orçamento participativo. Reflexões sobre a experiência de Porto Alegre. Porto Alegre: Tomo Editorial/ Rio de Janeiro: Observatório de Políticas Urbanas e Gestão Municipal (FASE/ IPPUR), 2. Aufl., 1999.

GENRO, Tarso; SOUZA, Ubiratan de. Quand les habitants gèrent vraiment leur ville. Le budget participatif: l'expérience de Porto Alegre au Brésil. Paris: Éditions Charles Leópold Mayer, 1998.

GRET, Marion; SINTOMER, Yves. Porto Alegre: L'espoir d'une autre démocratie. Paris: Éditions La Découverte et. Syros, 2002.

HABERMAS, Jürgen. Die Postnationale Konstellation: politische essays. Frankfurt/ Main: Suhrkamp, 1998.

. Jenseits des Nationalstaats?. In: BECK, Ulrich (Hrsg.).

Politik der Globalisierung. Frankfurt/ Main: Suhrkamp, 1998. S. 67 ff.

. Zur Legitimation Durch Menschenrechte. In:

BRUNKHORST, Hauke; NIESEN, Peter (Hrsg.). Das Recht der Republik. Frankfurt/ Main: Suhrkamp, 1999. S. 386 ff.

LUTZ-BACHMANN, Matthias. "Weltstaatlichkeit" und Menschenrechte nach dem Ende des überlieferten "Nationalstaats". In: BRUNKHORST, Haute; KÖHLER, Wolfgang; LUTZ-BACHMANN, Matthias (Hrsg.).

Recht auf Menschenrechte. Frankfurt/ Main: Suhrkamp, 1999. S. 199 ff. 
MAUS, Ingeborg. Basisdemokratische Aktivitäten und rechtsstaatliche Verfassung. In: KREUDER, Thomas (Hrsg.). Der orientierungslose Leviathan. Verfassungsdebatte, Funktion und Leistungsfähigkeit von Recht und Verfassung. Marburg: Schüren, 1992. S. 99 ff.

MÜLLER, Friedrich. Demokratie in der Defensive. Funktionelle Abnutzung - Soziale Exklusion - Globalisierung. Elemente einer Verfassungstheorie VII. Berlin: Duncker \& Humblot, 2001.

\section{. Demokratie zwischen Staatsrecht und Weltrecht:}

Nationale, staatlose und globale Formen menschenrechtsgestützer Globalisierung. Elemente einer Verfassungstheorie VIII. Berlin: Duncker \& Humblot, 2003.

NGUYEN, Van Nam. Die Rolle des Staates im Zeitalter der Globalisierung. Aachen: Shaker, 2002.

ROSAS, Allan. Globaler Konstitutionalismus, Menschenrechte und staatliche Souveränität. In: BRUNKHORST, Hauke; KETTNER, Matthias (Hrsg.). Globalisierung und Demokratie. Wirtschaft, Recht, Medien. Frankfurt/ Main: Suhrkamp, 2000. S. $151 \mathrm{ff}$.

SCHMALZ-BRUNS, Rainer. Deliberativer Supranationalismus. In: Zeitschrift für Internationale Beziehungen 6, 2 (1999). Baden-Baden. S. 185-244.

THÜRER, Daniel. "Citizenship" und Demokratieprinzip: "Föderative" Ausgestaltungen im Innerstaatlichen, Europäischen und Globalen Rechtskreis. In: BRUNKHORST, Hauke; KETTNER, Matthias (Hrsg.). Globalisierung und Demokratie. Wirtschaft, Recht, Medien. Frankfurt/ Main: Suhrkamp, 2000. S. $177 \mathrm{ff}$.

WEHLER, Hans-Ulrich. Nationalismus. Geschichte - Formen - Folgen. München: C.H. Beck Verlag, 2001. 